\title{
Superconductivity and charge density wave formation in lithium-intercalated $2 \mathrm{H}-\mathrm{TaS}_{2}$
}

\author{
Huanlong Liu, ${ }^{1}$ Shangxiong Huangfu, ${ }^{1,2}$ Xiaofu Zhang $\odot, 1,3,4,{ }^{*}$ Hai Lin, ${ }^{1}$ and Andreas Schilling $\odot{ }^{1}$ \\ ${ }^{1}$ Department of Physics, University of Zurich, Winterthurerstrasse 190, CH-8057 Zurich, Switzerland \\ ${ }^{2}$ Laboratory for High Performance Ceramics, Empa, Überlandstrasse 129, CH-8600 Dübendorf, Switzerland \\ ${ }^{3}$ State Key Laboratory of Functional Materials for Informatics, Shanghai Institute of Microsystem and Information Technology, \\ Chinese Academy of Sciences (CAS), Shanghai 200050, China \\ ${ }^{4}$ CAS Center for Excellence in Superconducting Electronics, Shanghai 200050, China
}

(Received 5 May 2021; revised 16 July 2021; accepted 10 August 2021; published 25 August 2021)

\begin{abstract}
We systematically investigated the superconducting properties and the interplay between charge density waves (CDWs) and superconductivity (SC) in lithium-intercalated $2 \mathrm{H}-\mathrm{TaS}_{2}$. By gradually increasing the lithium content $x$, the CDW formation temperature is continuously suppressed, and the onset temperature of SC is increased with a maximum transition temperature $T_{c}=3.5 \mathrm{~K}$ for $x=0.096$. The bulk nature of SC is confirmed by a superconducting shielding fraction of the order of unity for this composition. The electronic contribution to the specific heat and Hall resistivity data demonstrates that the CDW weakens with lithium intercalation, thereby indirectly increasing the carrier density and boosting SC. While the sign of the charge carriers in undoped $2 \mathrm{H}-\mathrm{TaS}_{2}$ changes from electronlike to hole type near the CDW formation temperature $\sim 75 \mathrm{~K}$, the lithium-intercalated $\mathrm{Li}_{x} \mathrm{TaS}_{2}$ shows predominantly hole-type carriers in the CDW phase even for very low lithium content.
\end{abstract}

DOI: 10.1103/PhysRevB.104.064511

\section{INTRODUCTION}

Layered transition-metal chalcogenides (TMDs) are typical quasi-two-dimensional electronic systems with multifarious phases [1-3], which show intriguing electronic and magnetic properties, including charge density waves (CDWs) and superconductivity (SC) [4-8]. SC and CDWs are two very different collective electronic phenomena, but they can coexist in the TMDs with the $2 H$ structure variant. Upon substituting $\mathrm{Se}$ by $\mathrm{S}$, for example, the superconducting critical temperature $\left(T_{c}\right)$ is enhanced from $2 \mathrm{H}-\mathrm{NbSe}_{2}\left(2 \mathrm{H}-\mathrm{TaSe}_{2}\right)$ through $2 H-\mathrm{NbS}_{2}\left(2 H-\mathrm{TaS}_{2}\right)$ while weakening the CDW state [4,9]. The results of angle-resolved photoemission spectroscopy experiments demonstrate that these two orders (CDWs and SC) in the above TMDs may be cooperative phenomena rather than competitive $[10,11]$. By contrast, it is usually found that the increase of $T_{c}$ is accompanied by the disappearance of CDWs for intercalated $2 H-\mathrm{TaS}_{2}$ [12-14]. The interplay between the superconducting and CDW states has also been investigated by high-pressure experiments on bulk $2 \mathrm{H}-\mathrm{TaS}_{2}$ $[15,16]$. The $T_{c}$ is enhanced dramatically to a maximal value, and the CDW is weakened and collapses at a critical pressure. At the same time, the electron-phonon coupling strength drastically decreases, and the electron density of states at the Fermi level suddenly increases beyond this critical pressure [17]. Increasing the pressure even further, $T_{c}$ reaches a maximum. Thus, the simple picture of the two orders in competition may fall too short. Near the collapse of the CDW, a quantum critical point is approached, and the associated

\footnotetext{
"zhangxf@mail.sim.ac.cn
}

quantum fluctuations may even enhance SC [16], which is reminiscent of the situation in the high-temperature superconductor $\mathrm{YBa}_{2} \mathrm{Cu}_{3} \mathrm{O}_{6+\delta}$ upon hole doping [18]. Therefore, the understanding of such spontaneous charge orders in two dimensions is essential to reveal the nature of SC in layered materials $[19,20]$.

$2 H-\mathrm{TaS}_{2}$ is a typical TMD material with coexisting CDWs and SC. Stoichiometric $2 H-\mathrm{TaS}_{2}$ is reported to show an inplane CDW formation at $\sim 75 \mathrm{~K}$, as well as a transition to $\mathrm{SC}$ at $T_{c} \sim 0.8 \mathrm{~K}[21,22]$. The $2 \mathrm{H}-\mathrm{TaS}_{2}$ (pyridine) $)_{1 / 2}$, as the first organic intercalation compound, shows an enhanced $T_{c}$ up to $3.5 \mathrm{~K}$ [23]. To further increase $T_{c}$, various alkali-metal or transition-metal atoms have been introduced into the structure to modify the electronic states of $2 \mathrm{H}-\mathrm{TaS}_{2}$ by doping or intercalation [24-27]. The superconducting transition temperature in $2 H-\mathrm{Cu}_{x} \mathrm{TaS}_{2}$, for example, is found to increase with copper intercalation [26], while the CDW is gradually weakened until it disappears at the maximum value of $T_{c}$, which is a very common feature of some intercalated $\mathrm{TaS}_{2}$ or other two-dimensional materials. However, there have still been only a few systematic studies on the intercalation of alkali metals and the resulting induced $\mathrm{SC}$ and suppressed $\mathrm{CDW}$ state in $2 \mathrm{H}-\mathrm{TaS}_{2}$. The sodium intercalated $2 \mathrm{H}-\mathrm{TaS}_{2}$ $\left(2 H-\mathrm{Na}_{x} \mathrm{TaS}_{2}\right)$ shows superconducting transitions with $T_{c}$ of $2.5 \mathrm{~K}$ in $\mathrm{Na}_{0.05} \mathrm{TaS}_{2}$ and $4.4 \mathrm{~K}$ in $2 \mathrm{H}-\mathrm{Na}_{0.1} \mathrm{TaS}_{2}[14,25]$. The CDW state is weakened, which has been proven by the anisotropy of resistivity and spectroscopic signatures on sodium intercalation [14,28]. Additionally, there are reports of lithium-intercalated $\mathrm{TaS}_{2}$ by solid-phase reaction or using $n$-butyllithium solution soaking strategies [24]. The corresponding $T_{c}$ can reach up to $4.5 \mathrm{~K}$, where the CDW state fully disappears. In general, the CDW phase and its transition are 

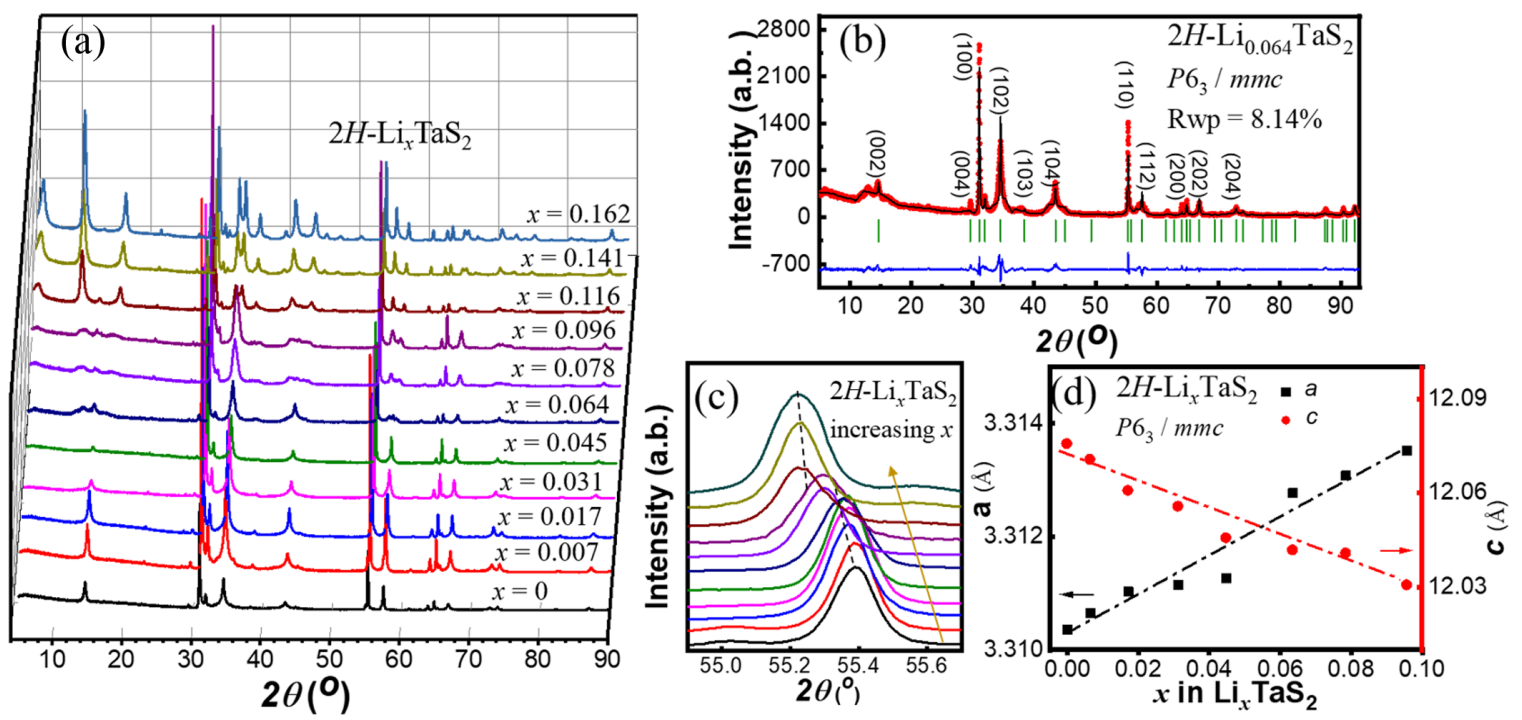

FIG. 1. (a) The powder x-ray diffraction (PXRD) pattern at ambient temperature for all samples of $2 H$-Li $\mathrm{T}_{x} \mathrm{TaS}_{2}(0 \leqslant x \leqslant 0.162)$. (b) The PXRD pattern of $2 \mathrm{H}$ - $\mathrm{Li}_{0.064} \mathrm{TaS}_{2}$. The red dots are the observed data, while the black solid line represents the calculated intensities. The bottom blue solid line is the difference between the observed and calculated intensities. (c) The enlarged (110) reflections with increasing $x$, indicating the variation of cell parameters. (d) The change of the cell parameter for $2 H$ - $\mathrm{Li}_{x} \mathrm{TaS}_{2}$ samples $(0 \leqslant x \leqslant 0.096)$.

not only related to the change of the Fermi level in the Ta- $5 d$ derived electron band upon alkali intercalation, but they are also affected by the superlattice formation due to interlayered alkali metal in $1 T-\mathrm{TaS}_{2}$ [29]. Therefore, the details of the effects of alkali metal intercalation on SC and CDWs are not yet fully understood.

To further clarify the nature of these two electronic states in intercalated $2 \mathrm{H}-\mathrm{TaS}_{2}$, we performed a systematic investigation on lithium-intercalated $2 H-\mathrm{TaS}_{2}$ including magnetization, heat-capacity, and transport measurements. The lithium was chosen because the valence electrons are not expected to participate in chemical bonds. Moreover, the lithium atom is only weakly paramagnetic and is not expected to be detrimental to SC. We find that the $T_{c}$ in $2 H-\mathrm{Li}_{x} \mathrm{TaS}_{2}$ first increases with lithium intercalation, in which the CDW state is concurrently suppressed. As the lithium content exceeds $\approx 10 \%$, the $T_{c}$ moderately decreases, which is associated with a structural change.

\section{EXPERIMENTS}

Polycrystalline $2 H-\mathrm{Li}_{x} \mathrm{TaS}_{2}$ samples were synthesized by solid-state reaction methods. Stoichiometric amounts of raw materials $\left(99.9 \% \mathrm{Li}_{2} \mathrm{~S}, 99.99 \% \mathrm{Ta}\right.$, and $99.9 \% \mathrm{~S}$ powders $)$ were mixed, ground, pressed into tablets, and sealed in evacuated silica tubes. The tubes were then loaded into a muffle furnace and annealed at $800^{\circ} \mathrm{C}$ for $12 \mathrm{~h}$. Finally, they were cooled down to room temperature along with the cooling of the furnace.

Single crystals for $x=0$ and 0.007 were obtained by extending the annealing time. The tubes were heated slowly up to $800^{\circ} \mathrm{C}$, sintered for $72 \mathrm{~h}$, and then slowly cooled down to $600{ }^{\circ} \mathrm{C}$ with a cooling rate of $2{ }^{\circ} \mathrm{C} / \mathrm{h}$. Finally, the tubes were cooled down in the furnace by autoswitching off the power.
The powder x-ray diffraction (PXRD) data of all the samples were collected by using a Stoe STADIP diffractometer at room temperature $\left(\mathrm{Cu} \mathrm{K}_{\alpha 1}\right.$ radiation, $\left.\lambda=1.54051 \AA\right)$. The inductively coupled plasma mass spectrometry (ICP-MS) measurements were performed with an Agilent QQQ 8800 Triple quad ICP-MS spectrometer. The transport measurements were performed with a Physical Property Measurement System (PPMS, Quantum Design Inc.) and a standard fourprobe technique. The heat capacity was measured with the heat-capacity option of the PPMS. The magnetic properties were studied in a Magnetic Properties Measurement System (MPMS 3 from Quantum Design Inc.)

\section{RESULTS AND DISCUSSION}

The influence of lithium intercalation on the structure of $2 H-\mathrm{TaS}_{2}$ is shown in Fig. 1. The identity and phase purity of the samples were determined by PXRD. As we can see in Fig. 1(a), the diffraction patterns of all samples of lithiumintercalated $2 H-\mathrm{TaS}_{2}$ solid solutions can be well fitted to the $2 \mathrm{H}$-type structures $\left(\mathrm{PG}_{3} / \mathrm{mmc}\right)$ for lithium content below $x=0.096$. The Rietveld refinement for $2 H-\mathrm{Li}_{0.064} \mathrm{TaS}_{2}$ is shown in Fig. 1(b) as an example, demonstrating high phase purity. The lithium content of these $2 H-\mathrm{Li}_{x} \mathrm{TaS}_{2}$ powders have been measured by ICP-MS. The slight systematic variation of the (110) peaks with increasing lithium content $x$ is shown in Fig. 1(c). For $x \leqslant 0.096$, the cell parameters of $a$ and $c$ show only small but systematic changes [Fig. 2(d)], resulting in a shrinking of the unit cell volume. The $c$ axis shows a regular decrease, which is like the trend observed in sodium-intercalated $\mathrm{NbS}_{2}$ and $2 H-\mathrm{TaS}_{2}$ [14,30]. This can be taken as evidence that the lithium atoms are not substituting Ta atoms but are intercalated into the interlayer regions of $2 \mathrm{H}-\mathrm{TaS}_{2}$. As the lithium content is increased further beyond 0.096, the crystal structure changes. The distance between the 

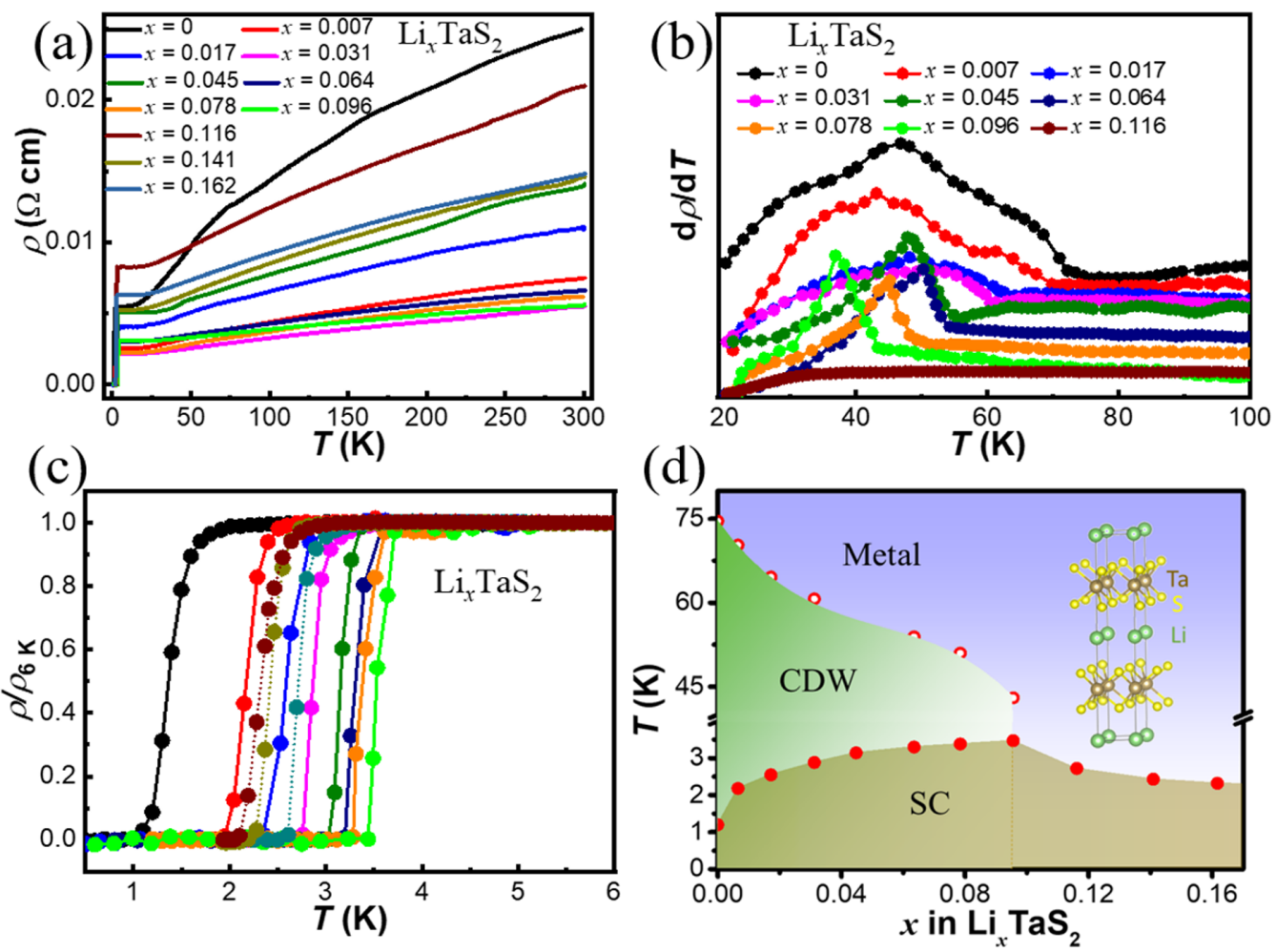

FIG. 2. The physical properties for all the samples of nominal composition $2 H-\mathrm{Li}_{x} \mathrm{TaS}_{2}(0 \leqslant x \leqslant 0.162)$. (a) Resistivity in a temperature range between 0.5 and $300 \mathrm{~K}$. (b) Temperature dependence of derivative of resistance, ranging from 20 to $100 \mathrm{~K}$. Some curves have been offset for clarity. The data for $x=0.141$ and 0.162 are like those for $x=0.116$ and are therefore not shown. (c) Normalized resistivity $\rho / \rho_{(6 \mathrm{~K})}$, between 0.5 and $6 \mathrm{~K}$. (d) The electronic phase diagram of $\mathrm{Li}_{x} \mathrm{TaS}_{2}$. Open circles represent the charge density wave (CDW) transition temperature, and the filled circles correspond to the superconducting transition temperature. Inset: $\mathrm{Crystal}$ structure of $2 \mathrm{H}$ - $\mathrm{Li}_{x} \mathrm{TaS}_{2}$.

two adjacent layers can be determined to $\sim 4.4 \AA$, which is much larger than for $\mathrm{Li}_{x} \mathrm{TaS}_{2}$ with $x<0.096(\sim 3.4 \AA)$. It is therefore unreasonable to assume that, at high lithium content, only lithium ions exist between the layers. As the samples of $\mathrm{Li}_{x} \mathrm{TaS}_{2}$ are air sensitive, it has been speculated that trace amounts of moisture enter the interlayer regions of $\mathrm{TaS}_{2}$ for chelation with lithium ions due to the unstable chemical nature of lithium, forming $\mathrm{Li}_{x}\left(\mathrm{H}_{2} \mathrm{O}\right)_{y} \mathrm{TaS}_{2}$ [31,32]. We could indeed verify this conjecture for $x \geqslant 0.22$ by a Rietveld refinement of corresponding PXRD data.

To investigate the effects of lithium intercalation on the CDW state and SC, we have performed comprehensive transport measurements. Figure 2(a) shows the temperature dependence of the resistivity for the low-lithium intercalation samples for temperatures ranging from 0.5 to 300 $\mathrm{K}$. In the normal state, the resistivity decreases with temperature, showing a weakly metallic behavior. The parent $2 \mathrm{H}-\mathrm{TaS}_{2}$ exhibits a CDW phase transition at a temperature $\sim 75 \mathrm{~K}$, which is consistent with corresponding literature values $[14,15]$, demonstrating the high quality of our compounds. The evolution of the CDW transition temperature with lithium intercalation was inferred from the maxima in the temperature derivative of the resistance $d \rho(T) / d T$, and it turns out to be strongly reduced with increasing lithium content. As we can see in Fig. 2(b), the CDW transition temperature is effectively suppressed by the lithium intercalation from $75 \mathrm{~K}$ for $x=0$ to $41 \mathrm{~K}$ for $x=0.096$ and vanishes with a further increase of the lithium content above $x=0.1$. Figure 2(c) shows the transition to $\mathrm{SC}$ for $\mathrm{Li}_{x} \mathrm{TaS}_{2}$ on an expanded scale. The undoped $2 H-\mathrm{TaS}_{2}$ shows a transition at $T_{c} \approx 1.2 \mathrm{~K}\left(T_{c}\right.$ defined by a $50 \%$ criterion), which is like the reported results of $T_{c}$ with zero resistance $\sim 0.8 \mathrm{~K}$ [33]. Upon lithium intercalation, $T_{c}$ increases, and the CDW ordering temperature $T_{\mathrm{CDW}}$ decreases with increasing $x$, demonstrating that there is a correlation between coexisting SC and CDW phases. The $T_{c}$ reaches a maximum of $\approx 3.5 \mathrm{~K}$ for $x=0.096$ and then decreases for higher lithium content, which we attribute to the formation of $\mathrm{Li}_{x}\left(\mathrm{H}_{2} \mathrm{O}\right)_{y}$ as a new intercalator, as we have discussed above. The resulting phase diagram is shown in Fig. 2(d), which is like the electronic phase diagram of $2 H-\mathrm{TaSe}_{2-x} \mathrm{~S}_{x}$ [34].

Both CDWs and SC are closely related to the conduction electrons. To further reveal the electronic effect of lithium intercalation on CDWs and SC, the Hall resistivity has been measured to estimate the carrier type and density in $2 H-\mathrm{Li}_{x} \mathrm{TaS}_{2}$. As the samples with large lithium content became quite unstable, it was difficult to obtain enough large crystals. We therefore only measured the Hall resistivity on single crystals with low lithium content, as shown in Figs. 3(a) and 3 (b) for undoped $2 H-\mathrm{TaS}_{2}$ and $2 \mathrm{H}-\mathrm{Li}_{0.007} \mathrm{TaS}_{2}$ crystals, respectively. The magnetic field-dependent Hall resistivity $\rho_{x y}$ shows an ideal linear dependence, allowing us to calculate the carrier density in a simple single-band model. These results are summarized in Fig. 3(c). The temperaturedependent carrier densities of undoped $2 \mathrm{H}-\mathrm{TaS}_{2}$ crystals show 

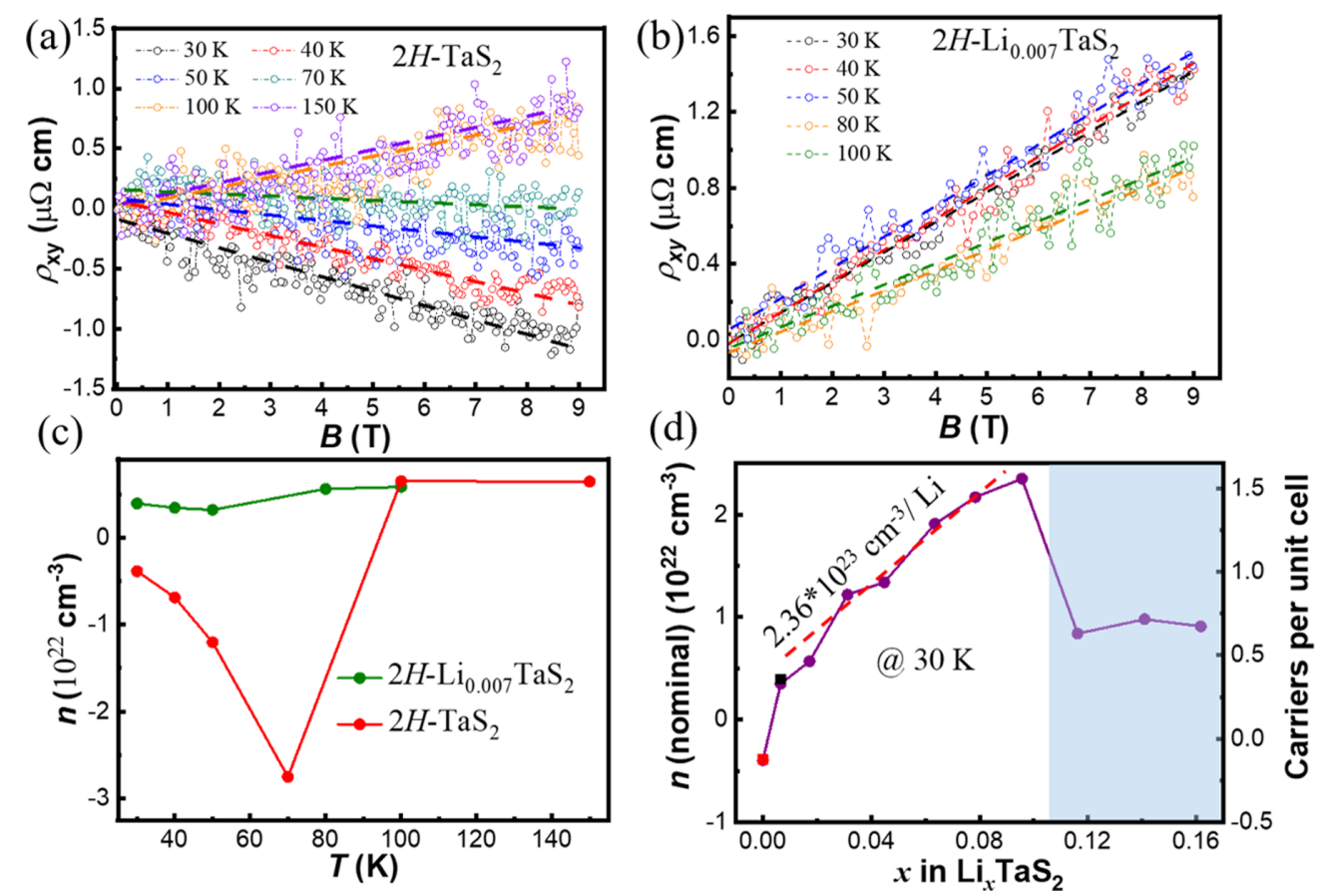

FIG. 3. (a) Magnetic field-dependent Hall resistance of $2 H-\mathrm{TaS}_{2}$ and (b) $2 H-\mathrm{Li}_{0.007} \mathrm{TaS}_{2}$ crystals. (c) The corresponding temperaturedependent carrier densities of $2 \mathrm{H}-\mathrm{TaS}_{2}$ and $2 \mathrm{H}-\mathrm{Li}_{0.007} \mathrm{TaS}_{2}$ single crystals. (d) Nominal charge carrier density and corresponding carriers per unit cell as obtained at $T=30 \mathrm{~K}$ from $2 H-\mathrm{Li}_{x} \mathrm{TaS}_{2}$ polycrystalline samples. The dashed line is linear fit to these data for $0<x<0.1$. The red and the green data points are from the data for single crystals shown in Fig. 3(c).

a sudden change both in the magnitude and sign as the temperature approaches $T_{\mathrm{CDW}}$, which is like reports on $2 \mathrm{H}-\mathrm{TaS}_{2}$, $\mathrm{NbSe}_{2}$, and $\mathrm{YBa}_{2} \mathrm{Cu}_{3} \mathrm{O}_{6+\delta}$ [27,35-37]. Two kinds of charge carriers, electrons and holes, are dominating the transport behavior below and above the CDW phase transition, respectively. For the $2 H-\mathrm{Li}_{0.007} \mathrm{TaS}_{2}$ crystals, however, the positive Hall resistivity indicates that the dominant carriers are holes in this system. An associated comparably small change in the carrier density between 60 and $80 \mathrm{~K}$ also confirms the CDW transition, in a similar way as it has been observed in $2 H-\mathrm{Cu}_{0.03} \mathrm{TaS}_{2}$ [38] and $2 H-\mathrm{In}_{0.5} \mathrm{TaS}_{2}$ [39]. The carrier density measured on single-crystalline $2 H-\mathrm{Li}_{0.007} \mathrm{TaS}_{2}$ is somewhat larger $\left(\approx 3.95 \times 10^{21}\right.$ holes $\mathrm{cm}^{-3}$ at $\left.T=30 \mathrm{~K}\right)$ than the undoped $2 \mathrm{H}-\mathrm{TaS}_{2}\left(\approx 3.86 \times 10^{21}\right.$ electrons $\mathrm{cm}^{-3}$ at $T=$ $30 \mathrm{~K}$ ), which would correspond to $\approx 0.23$ and 0.22 charge carriers per unit cell, respectively. In the CDW state above $T_{\mathrm{CDW}}, n$ is of the order of $\approx 5 \times 10^{21} \mathrm{~cm}^{-3}$ for both compositions, which corresponds to $\approx 0.3$ holelike carriers per unit cell. To obtain a systematic trend as a function of lithium content $x$, we also performed Hall effect measurements on polycrystalline samples at $T=30 \mathrm{~K}$. The absolute values of the corresponding numbers must be taken with the reservation that Hall measurements on polycrystals can be affected by anisotropy [40] and grain boundary effects [41]. Nevertheless, as all the samples were prepared in a similar manner, we can still identify a clear trend. The data shown in Fig. 3(d) indicate that there is an almost linear increase in $n$ as a function of lithium content up to $x=0.096$ and a sudden drop for larger values of $x$, which is not unexpected due to the changes in the crystal structure, as discussed above. Using these estimates based on the linear fitting [see Fig. 3(d)], we find that the hypothetical intercalation of one lithium atom would correspond to a change in $n$ by $\approx 14$ carriers per formula unit. Therefore, the lithium intercalation does not primarily act as a mechanism for carrier doping but most probably leads to a weakening of the CDW state, which indirectly results in an increase of the density of mobile charge carriers with a resulting boost to $\mathrm{SC}$.

To study the physical properties of lithium-intercalated $2 \mathrm{H}-\mathrm{TaS}_{2}$ superconductors in more detail, we have chosen to compare intrinsic $2 H-\mathrm{TaS}_{2}\left(T_{c} \approx 1.2 \mathrm{~K}\right)$ with $2 H-\mathrm{Li}_{0.096} \mathrm{TaS}_{2}$ (with a maximum $T_{c} \approx 3.5 \mathrm{~K}$ ). Detailed measurements of the field dependence of the resistive transition to SC and the magnetization are presented in Fig. 4. The effect of applying a magnetic field on $T_{c}$ for the $x=0$ and 0.096 samples are shown in Figs. 4(a) and 4(b), respectively. As expected, $T_{c}$ is gradually suppressed, and the width of the superconducting transition increases as the magnetic field is increased. The resulting temperature dependences of the upper critical fields $\mu_{0} H_{c 2}(T)$ are shown in Fig. 4(c). The extrapolated slopes are $d H_{c 2} / d T=-1.41$ and $-1.78 \mathrm{~T} / \mathrm{K}$ for $x=0$ and 0.096 , respectively. The upper critical fields at zero temperature $\mu_{0} H_{c 2}(0)$ can be estimated using the Werthamer-HelfandHohenberg (WHH) approximation in the dirty limit [25,42]:

$$
\mu_{0} H_{c 2}^{\mathrm{WHH}}(0)=-0.69 T_{c}\left(\frac{d H_{c 2}}{d T}\right)_{T=T_{c}},
$$

to $\mu_{0} H_{c 2}^{\mathrm{WHH}}(0) \approx 1.17 \mathrm{~T}$ for $x=0$, and $\mu_{0} H_{c 2}^{\mathrm{WHH}}(0) \approx 4.24 \mathrm{~T}$ for $x=0.096$, respectively. From $\mu_{0} H_{c 2}=\Phi_{0} / 2 \pi \xi(0)^{2}$, we can estimate the corresponding superconducting coherence lengths $\xi(0)=16.8$ and $8.8 \mathrm{~nm}$, respectively. 

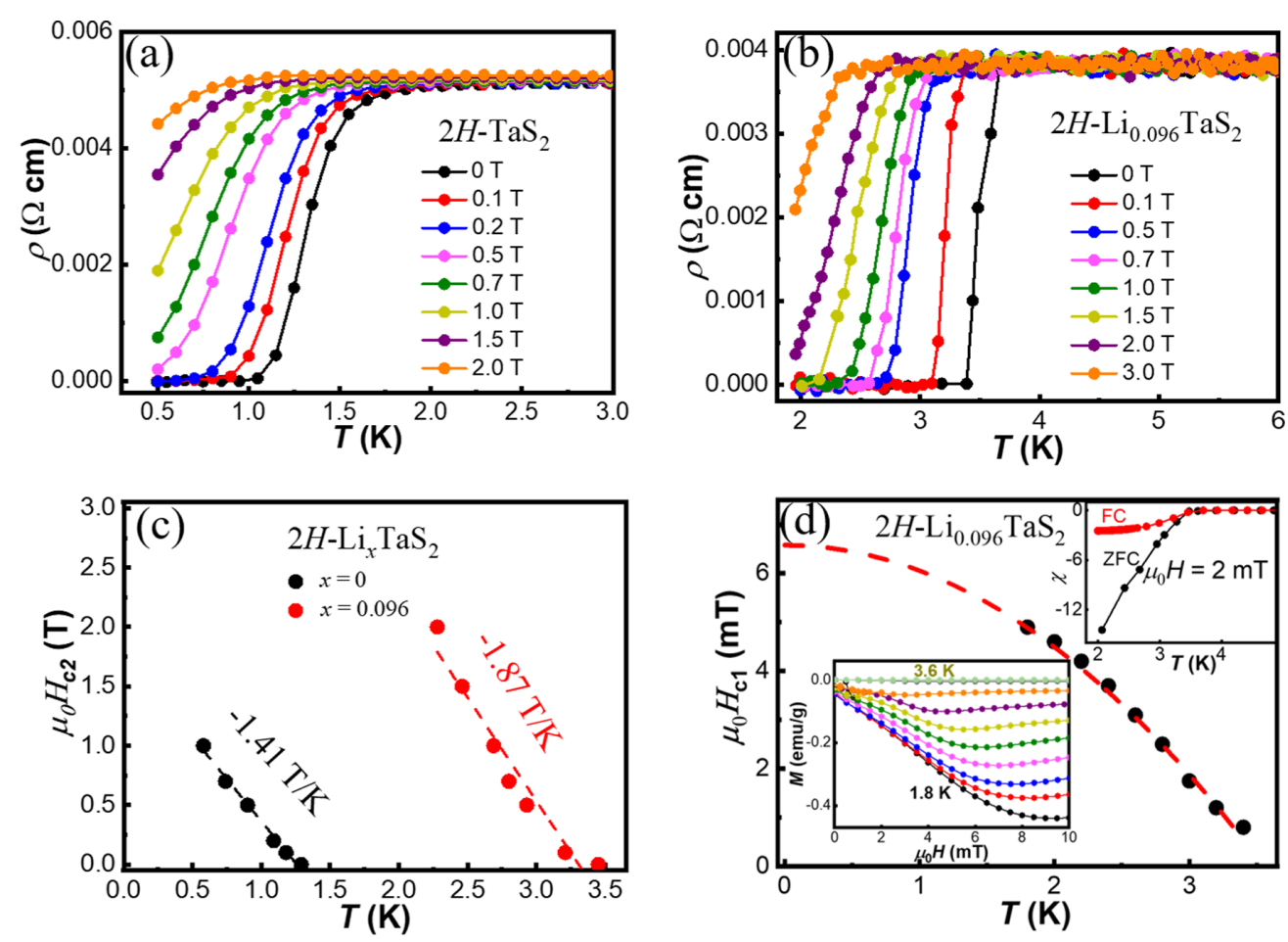

FIG. 4. (a) and (b) Field-dependent resistivity measurements for the samples $x=0$ and 0.096 . (c) The dashed lines indicate the extrapolated slopes $d H_{c 2} / d T$ used for the Werthamer-Helfand-Hohenberg (WHH) approximation. (d) The temperature dependence of the lower critical field $H_{c 1}$ for $x=0.096$. Lower inset: the zero-field-cooled (ZFC) field dependence of the magnetization $M(H)$ for temperatures between 1.8 and 3.6 $\mathrm{K}$ (in $0.2 \mathrm{~K}$ steps) in magnetic fields $\mu_{0} H$ between 0 and $15 \mathrm{mT}$, and upper inset: ZFC and field-cooled (FC) magnetic susceptibility.

The zero-field-cooled (ZFC) field dependence of the magnetization $M(H)$ for temperatures between 1.8 and $3.6 \mathrm{~K}$ is shown in Fig. 4(d) for the $2 H-\mathrm{Li}_{0.096} \mathrm{TaS}_{2}$ sample, exhibiting typical type-II superconducting behavior. The ZFC and the field-cooled (FC) magnetic susceptibilities measured in $2 \mathrm{mT}$ are shown in the lower inset of Fig. 4(d). A large superconducting shielding fraction of $\sim 120 \%$, as determined from comparing the measured magnetization with theoretical expectations, is an indication of the bulk nature of SC, thereby confirming complete magnetic flux expulsion, as required by the Meissner-Ochsenfeld effect (an overestimated value $>100 \%$ is most probably caused by demagnetization effects). By defining the lower critical field $H_{c 1}$ as the minimum on the $M(H)$ curves, its temperature dependence can be well fitted using an empirical formula [43]:

$$
\mu_{0} H_{c 1}(T)=\mu_{0} H_{c 1}(0)\left[1-\left(\frac{T}{T_{c}}\right)^{2}\right]
$$

A resulting estimate of the corresponding lower critical field is $\mu_{0} H_{c 1}(0) \approx 6 \mathrm{mT}$. Together with $\mu_{0} H_{c 1}(T)=$ $\frac{\Phi_{0}}{4 \pi \lambda^{2}} \ln \frac{\lambda}{\xi}$, we obtain an estimate for the London penetration depth $\lambda(0) \simeq 310 \mathrm{~nm}$ and $\kappa=\frac{\lambda(0)}{\xi(0)} \simeq 35.2$, confirming that the material is a strongly type-II superconductor.

The low-temperature specific heats of the $2 H-\mathrm{Li}_{x} \mathrm{TaS}_{2}$ samples $(x=0.045,0.064$, and 0.096) are shown in Fig. 5. As expected, the specific heat data show a peak at $T_{c} \approx 2.9$, 3.3 , and $3.5 \mathrm{~K}$, respectively. These results are consistent with the data from our transport measurements. The normal state specific heat can be fitted by a standard expression at low temperatures:

$$
\frac{C_{p}}{T}=\gamma+\beta T^{2},
$$

where $\gamma$ is the Sommerfeld constant, which is proportional to the electron density of states $D\left(E_{F}\right)$ at the Fermi level. The fitted values of $\beta$ for all selected samples are $\sim 0.4 \mathrm{~mJ} / \mathrm{mol} \mathrm{K}^{4}$ (see Table I), as reported for $\mathrm{Cu}_{x} \mathrm{TaS}_{2}$ and $\mathrm{Cu}_{x} \mathrm{TiSe}_{2}$ [26,44], corresponding to a Debye temperature of $\sim 244 \mathrm{~K}$, which we have calculated from the corresponding three-dimensional lattice Debye model. The resulting electronic contributions to the

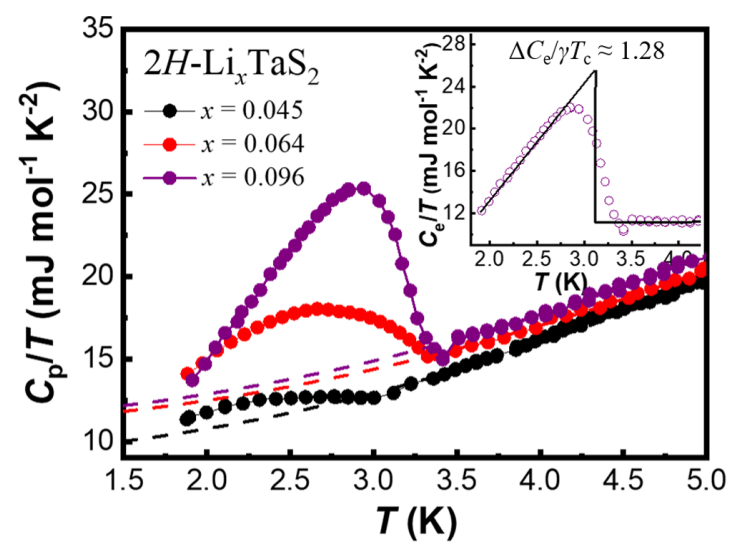

FIG. 5. Specific-heat $C / T$ for $2 H-\mathrm{Li}_{x} \mathrm{TaS}_{2}$ with different $x$ values. Right inset: The solid line shows an entropy-conserving construction to obtain $\Delta C_{e} / \gamma T_{c}$ for an optimally intercalated $2 H-\mathrm{Li}_{0.096} \mathrm{TaS}_{2}$ sample. 
TABLE I. Electronic $(\gamma)$ and lattice $(\beta)$ contributions according to a fit to Eq. (3). We obtained the calculated values $\gamma_{c}$ from the freeelectron model using the measured carrier densities $n$.

\begin{tabular}{|c|c|c|c|c|c|}
\hline 0 & 0.35 [45] & $3.5 \times 10^{21}$ & 0.88 & $8.5[45,46]$ & 9.66 \\
\hline 0.064 & $0.39(1)$ & $1.05 \times 10^{22}$ & 1.27 & 10.9 & 8.59 \\
\hline 0.096 & $0.40(2)$ & $1.2 \times 10^{22}$ & 1.33 & 11.2 & 8.35 \\
\hline
\end{tabular}

specific heat for these lithium-intercalated samples increases with lithium content, and they are slightly larger than that of the parent compound $2 \mathrm{H}-\mathrm{TaS}_{2}\left(\gamma=8.5 \mathrm{~mJ} / \mathrm{mol} \mathrm{K}^{2}\right)[45,46]$. These results demonstrate that the lithium intercalation increases, along with the charge carrier density $n$, also the electron density of states at the Fermi level [47]. We state here that the measured values for $\gamma$ of the order of $10 \mathrm{~mJ} / \mathrm{mol} \mathrm{K}^{-2}$ are far larger than one can expect from a simple free-electron model. Assuming one charge carrier per unit cell, we obtain with $\gamma_{c}=\pi^{2} k_{B}^{2} D\left(E_{F}\right) / 3$ and $D\left(E_{F}\right)=(3 n / \pi)^{1 / 3} m_{e} /\left(\pi \hbar^{2}\right)$ a calculated value $\gamma_{c}$ of the order of $1.5 \mathrm{~mJ} / \mathrm{mol} \mathrm{K}^{-2}$ only, which may hint at an enhanced effective mass in superconducting $2 H-\mathrm{Li}_{x} \mathrm{TaS}_{2}$ (see Table I). Here, $k_{B}$ is the Boltzmann constant, $m_{e}$ the electron mass, and $\hbar$ the reduced Planck constant. The corresponding effective mass enhancements estimated from the measured carrier densities and the specific heat data are all of the order of $\approx 8-9$ (see Table I). The right inset of Fig. 5 shows the discontinuity in the electronic specific heat $\left(C_{e} / T_{c}\right)$ at the superconducting transition with the phonon contribution subtracted and with a Bardeen-Cooper-Schrieffer (BCS) entropy-conserving construction. The obtained ratio $\Delta C_{e} / \gamma T_{c}=1.28$ is very close to the standard BCS value 1.43, thereby qualifying $2 \mathrm{H}-\mathrm{Li}_{x} \mathrm{TaS}_{2}$ as weakly coupled superconductors [48].

\section{CONCLUSIONS}

Figure 2(d) summarizes the electronic phase diagram of $2 H-\mathrm{Li}_{x} \mathrm{TaS}_{2}$, showing the evolution of the superconducting, CDW, and metallic phases with varying lithium content $x$. The superconducting and CDW states are interrelated and coexist with each other. The lithium intercalation gradually enhances the superconducting transition temperature and weakens the CDW state. At $x \approx 0.096$, the CDW phase is fully suppressed, and $\mathrm{SC}$ reaches its maximum critical temperature $T_{c}=3.5 \mathrm{~K}$, with a fully developed discontinuity in the specific heat which is compatible with a weak-coupling scenario. The changes upon lithium intercalation are accompanied by an increase of the hole-type carrier density. However, the measured changes in the charge carrier densities are too large to be explained by doping alone. We therefore suggest that lithium intercalation leads primarily to a weakening of the CDW state, which then indirectly causes an increase of the density of mobile holetype charge carriers.

\section{ACKNOWLEDGMENT}

This paper was supported by the Schweizerische Nationalfonds zur Förderung der Wissenschaftlichen Forschung (Grants No. 20-175554 and No. 206021-150784).
[1] M. Chhowalla, H. S. Shin, G. Eda, L.-J. Li, K. P. Loh, and H. Zhang, Nat. Chem. 5, 263 (2013).

[2] B. Radisavljevic, A. Radenovic, J. Brivio, V. Giacometti, and A. Kis, Nat. Nanotechnol. 6, 147 (2011).

[3] Q. H. Wang, K. Kalantar-Zadeh, A. Kis, J. N. Coleman, and M. S. Strano, Nat. Nanotechnol. 7, 699 (2012).

[4] A. H. Castro Neto, Phys. Rev. Lett. 86, 4382 (2001).

[5] D. W. Shen, B. P. Xie, J. F. Zhao, L. X. Yang, L. Fang, J. Shi, R. H. He, D. H. Lu, H. H. Wen, and D. L. Feng, Phys. Rev. Lett. 99, 216404 (2007).

[6] J. A. W. Straquadine, F. Weber, S. Rosenkranz, A. H. Said, and I. R. Fisher, Phys. Rev. B 99, 235138 (2019).

[7] D. Shin, N. Tancogne-Dejean, J. Zhang, M. S. Okyay, A. Rubio, and N. Park, Phys. Rev. Lett. 126, 196406 (2021).

[8] A. Fang, A. G. Singh, J. A. W. Straquadine, I. R. Fisher, S. A. Kivelson, and A. Kapitulnik, Phys. Rev. Research 2, 043221 (2020).

[9] J. A. Wilson and A. D. Yoffe, Adv. Phys. 18, 193 (1969).

[10] T. Valla, A. V. Fedorov, P. D. Johnson, P. A. Glans, C. McGuinness, K. E. Smith, E. Y. Andrei, and H. Berger, Phys. Rev. Lett. 92, 086401 (2004).
[11] T. Valla, A. V. Fedorov, P. D. Johnson, J. Xue, K. E. Smith, and F. J. DiSalvo, Phys. Rev. Lett. 85, 4759 (2000).

[12] L. J. Li, X. D. Zhu, Y. P. Sun, H. C. Lei, B. S. Wang, S. B. Zhang, X. B. Zhu, Z. R. Yang, and W. H. Song, Physica C 470, 313 (2010).

[13] X. Zhu, Y. Sun, S. Zhang, J. Wang, L. Zou, L. E. Delong, X. Zhu, X. Luo, B. Wang, G. Li, Z. Yang, and W. Song, J. Phys. Condens. Matter 21, 145701 (2009).

[14] L. Fang, Y. Wang, P. Y. Zou, L. Tang, Z. Xu, H. Chen, C. Dong, L. Shan, and H. H. Wen, Phys. Rev. B 72, 014534 (2005).

[15] D. C. Freitas, P. Rodière, M. R. Osorio, E. Navarro-Moratalla, N. M. Nemes, V. G. Tissen, L. Cario, E. Coronado, M. GarcíaHernández, S. Vieira, M. Núñez-Regueiro, and H. Suderow, Phys. Rev. B 93, 184512 (2016).

[16] X.-M. Zhao, K. Zhang, Z.-Y. Cao, Z.-W. Zhao, V. V. Struzhkin, A. F. Goncharov, H.-K. Wang, A. G. Gavriliuk, H.-K. Mao, and X.-J. Chen, Phys. Rev. B 101, 134506 (2020).

[17] K. Zhang, H. Jiang, J. Yang, J. Zhang, Z. Zeng, X. Chen, and F. Su, Appl. Phys. Lett. 117, 101105 (2020). 
[18] B. J. Ramshaw, S. E. Sebastian, R. D. McDonald, J. Day, B. S. Tan, Z. Zhu, J. B. Betts, R. Liang, D. A. Bonn, W. N. Hardy, and N. Harrison, Science 348, 317 (2015).

[19] J. Chang, E. Blackburn, A. T. Holmes, N. B. Christensen, J. Larsen, J. Mesot, R. Liang, D. A. Bonn, W. N. Hardy, A. Watenphul, M. v. Zimmermann, E. M. Forgan, and S. M. Hayden, Nat. Phys. 8, 871 (2012).

[20] Y. I. Joe, X. M. Chen, P. Ghaemi, K. D. Finkelstein, G. A. de la Peña, Y. Gan, J. C. T. Lee, S. Yuan, J. Geck, G. J. MacDougall, T. C. Chiang, S. L. Cooper, E. Fradkin, and P. Abbamonte, Nat. Phys. 10, 421 (2014).

[21] L. F. Mattheiss, Phys. Rev. B 8, 3719 (1973).

[22] R. Delaplace, P. Molinie, and D. Jerome, J. Phys. Lett. 37, 13 (1976).

[23] F. R. Gamble, F. J. DiSalvo, R. A. Klemm, and T. H. Geballe, Science 168, 568 (1970).

[24] Y. Nozuyama and S. Iida, Jpn. J. Appl. Phys. 23, L319 (1984).

[25] A. Lerf, F. Sernetz, W. Biberacher, and R. Schöllhorn, Mater. Res. Bull. 14, 797 (1979).

[26] K. E. Wagner, E. Morosan, Y. S. Hor, J. Tao, Y. Zhu, T. Sanders, T. M. McQueen, H. W. Zandbergen, A. J. Williams, D. V. West, and R. J. Cava, Phys. Rev. B 78, 104520 (2008).

[27] N. Z. Wang, M. Z. Shi, C. Shang, F. B. Meng, L. K. Ma, X. G. Luo, and X. H. Chen, New J. Phys. 20, 023014 (2018).

[28] W. Z. Hu, G. Li, J. Yan, H. H. Wen, G. Wu, X. H. Chen, and N. L. Wang, Phys. Rev. B 76, 045103 (2007).

[29] C. Pettenkofer and W. Jaegermann, Phys. Rev. B 50, 8816 (1994).

[30] W. P. F. A. M. Omloo, and F. Jellinek, J. Less Common Met. 20, 121 (1970).

[31] D. C. Johnston, Mater. Res. Bull. 17, 13 (1982).

[32] D. C. Johnston and S. P. Frysinger, Phys. Rev. B 30, 980 (1984).

[33] S. Nagata, T. Aochi, T. Abe, S. Ebisu, T. Hagino, Y. Seki, and K. Tsutsumi, J. Phys. Chem. Solids 53, 1259 (1992).
[34] L. Li, X. Deng, Z. Wang, Y. Liu, M. Abeykoon, E. Dooryhee, A. Tomic, Y. Huang, J. B. Warren, E. S. Bozin, S. J. L. Billinge, Y. Sun, Y. Zhu, G. Kotliar, and C. Petrovic, npj Quantum Materials 2, 11 (2017).

[35] H. N. S. Lee, H. McKinzie, D. S. Tannhauser, and A. Wold, J. Appl. Phys. 40, 602 (1969).

[36] L.-j. Li, Z.-a. Xu, J.-q. Shen, L.-m. Qiu, and Z.-h. Gan, J. Phys. Condens. Matter 17, 493 (2005).

[37] P. M. C. Rourke, A. F. Bangura, C. Proust, J. Levallois, N. Doiron-Leyraud, D. LeBoeuf, L. Taillefer, S. Adachi, M. L. Sutherland, and N. E. Hussey, Phys. Rev. B 82, 020514(R) (2010).

[38] X. D. Zhu, Y. P. Sun, X. B. Zhu, X. Luo, B. S. Wang, G. Li, Z. R. Yang, W. H. Song, and J. M. Dai, J. Cryst. Growth 311, 218 (2008).

[39] Y. Li, Z. Wu, J. Zhou, K. Bu, C. Xu, L. Qiao, M. Li, H. Bai, J. Ma, Q. Tao, C. Cao, Y. Yin, and Z.-A. Xu, Phys. Rev. B 102, 224503 (2020).

[40] T. K. Xia and D. Stroud, Phys. Rev. B 37, 118 (1988).

[41] A. K. Ghosh, A. Rose, H. P. Maruska, D. J. Eustace, and T. Feng, Appl. Phys. Lett. 37, 544 (1980).

[42] F. von Rohr, R. Nesper, and A. Schilling, Phys. Rev. B 89, 094505 (2014).

[43] E. H. Brandt, Phys. Rev. B 68, 054506 (2003).

[44] E. Morosan, H. W. Zandbergen, B. S. Dennis, J. W. G. Bos, Y. Onose, T. Klimczuk, A. P. Ramirez, N. P. Ong, and R. J. Cava, Nat. Phys. 2, 544 (2006).

[45] M. Abdel-Hafiez, X. M. Zhao, A. A. Kordyuk, Y. W. Fang, B. Pan, Z. He, C. G. Duan, J. Zhao, and X. J. Chen, Sci. Rep. 6, 31824 (2016).

[46] M. H. Van Maaren, Phys. Lett. A 40, 353 (1972).

[47] J. Pan, C. Guo, C. Song, X. Lai, H. Li, W. Zhao, H. Zhang, G. Mu, K. Bu, T. Lin, X. Xie, M. Chen, and F. Huang, J. Am. Chem. Soc. 139, 4623 (2017).

[48] D. C. Johnston, Supercond. Sci. Technol. 26, 115011 (2013). 